\title{
Enabling Future Nanotomography and Nanofabrication with Crossbeam Technology
}

\author{
Ingo Schulmeyer, Martin Kienle
}

\section{Carl Zeiss Microscopy GmbH, Carl-Zeiss-Straße 22, 73447 Oberkochen, Germany}

A Focused Ion Beam ( FIB) combined in one instrument with a Field Emission Scanning Electron Microscope (FE-SEM) has become a powerful instrument for numerous Standard and cutting edge applications in Research and Industry. The FIB is mainly used to open up the third dimension to a SEM. The ion beam is not only able to cut slices of a samples surface for tomographic imaging, but can also be used to create new materials or functional structures with superior properties. With increasing application maturity also the demand for faster systems, complete detection, more precise structuring and a wider application range rises.

3D-imaging and -analytics allow a complete characterization of a samples volume. It is widely used in Materials and Life Sciences and allows better understanding of compound materials, brain tissues, electronic devices and other samples. To achieve representative information of a sample, the analyzed volume needs to be sufficiently large and the resolution has to be high. The voxel re solution in tomography is mainly limited by the thickness of the slices cut with the ion beam [1]. The Crossbeam 540 allows the thinnest slices down to $5 \mathrm{~nm}$ and below. To keep the thickness homogenous over thousands of slices, a long -term stable FIB comes along with a sophisticated Software solution including adaptive slice thickness tracking (Figure 1). In interaction with a charged particle beam, each material behaves different. We introduce solutions to process, image and analyze all kinds of samples, including charging, outgassing or dirty and even magnetic samples. For a comprehensive understanding of a sample, it is vital to combine the information of the different detector signals. We will discuss our latest developments in detector technology to improve the signals especially at low acceleration voltages and the acquisition time. Acquisition of up to 4 detector signals simultaneously provides not only the maximum information in a single shot, but also the best protection of the sample.

Preparation of samples for TEM or other imaging techniques is one of the main applications for modern FIB-SEM instruments. Not only the quality, thickness and homogeneity of the prepared specimen matters, but also an fast, easy and integrated workflow. The quality of the results in TEM and STEM depend not only on the instrument, but to a high degree on the analyzed specimen. We demonstrate our latest improvements in ion beam technology, manipulator control and application development that form a complete Multi-Site Sample preparation workflow for thinnest high-quality lamellas. A guided workflow allows fast milling and cut-out of the lamella. The unique $\mathrm{X}^{2}$ method combined with a FIB that performs excellently at low voltages provides homogenous TEM lamellas with $<10 \mathrm{~nm}$ thickness and minimum amorphous layer [2] (Figure 2).

Modern FIB-SEM systems cover not only the typical application range but are also used to host and integrate numerous instruments and components for advanced experiments. The Zeiss FIB-SEM allows integration of many components for In-Situ analysis like heating, cooling and tensile stages, SIMS, EBIC and CL, different manipulators, e.g. for probing and liftout, a Laser option for micropatterning or fast removal of large amounts of material and many more. A modern API interface that opens the complete parameter space to the user allows customization and automation of complex workflows. 


\section{References:}

[1] L. Holzer and M. Cantoni, Review of FIB-tomography, Nanofabrication Using Focused Ion and Electron Beams: Principles and Applications (2011), p. $410 \mathrm{ff}$

[2] L. Lechner, J. Biskupek and U. Kaiser, Improved Focused Ion Beam Target Preparation of (S)TEM Specimen - A Method for Obtaining Ultrathin Lamellae, Microscopy and Microanalysis 18 (2012), p. 379-384

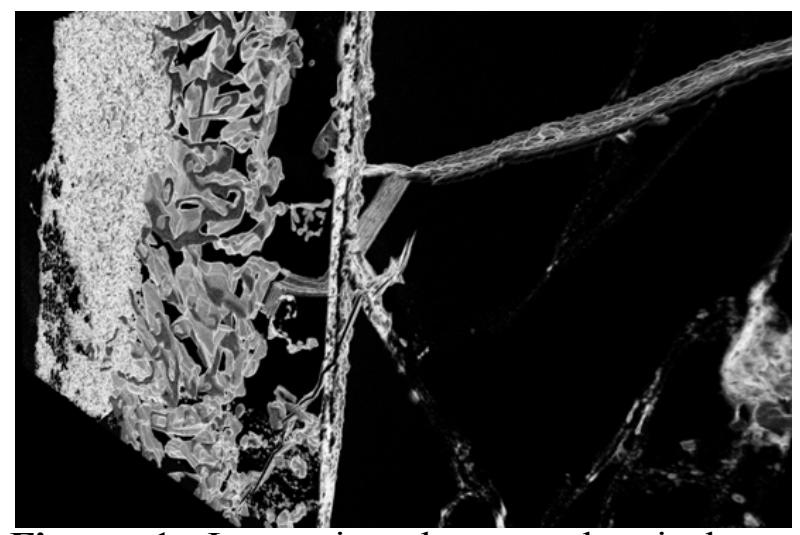

Figure 1. Innovative the rme-electrical generator transforming exhaust heat into eletrical power $\left(\mathrm{Mg}_{2} \mathrm{Sn}-\mathrm{Mg}_{2} \mathrm{Si}\right) .3 \mathrm{D}$ stack was acquired on Crossbeam running ATLAS3D. 8nm Voxel Size. The diffusion zone thickness varies between 10 to $25 \mu \mathrm{m}$
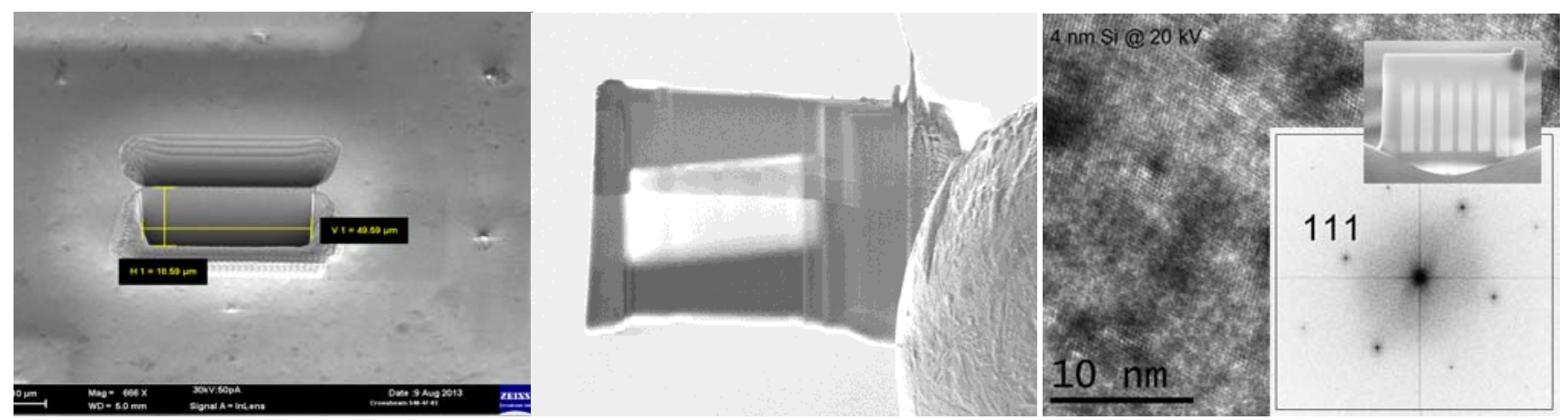

Figure 2. large $50 \mu \mathrm{m}$ x $20 \mu \mathrm{m}$ TEM lamella prepared automatically in $25 \mathrm{~min}$ (left). Lamella thinned using the $\mathrm{X}^{2}$-method (centre). HRTEM image of a lamella demonstrating atomic resolution at $20 \mathrm{kV}$. The spotty contrast variations in the image are caused by strong dynamic diffraction contrast that cannot be avoided at $20 \mathrm{kV}[2]$ (right). 